\title{
Aproximación a la traducción DE UNIDADES PAREMIOLÓGICAS DEL ESPAÑOL A LA LENGUA DE SIGNOS ESPAÑOLA
}

\author{
An Approach to the Translation of Paremiological Units from Spanish \\ into The Spanish Sign LANguage
}

UNE APPROCHE DE LA TRADUCTION DES UNITÉS PARÉMIOLOGIQUES DE L'ESPAGNOL À LA LANGUE DES SIGNES ESPAgNOLE

\section{ABORDAGEM À TRADUÇÃo DE UNIDADES PAREMIOLÓGICAS DO ESPANHOL PARA A LÍNGUA GESTUAL ESPANHOLA}

\author{
Rayco H. González-Montesino \\ Profesor, Universidad Rey Juan \\ Carlos, Campus de Vicálvaro, \\ Madrid, España. \\ raycoh.gonzalez@uric.es \\ https://orcid. \\ org/0000-0002-6830-3951
}

\begin{abstract}
RESUMEN
Las paremias son parte del acervo cultural de cada pueblo, tienen un sentido idiomático y metafórico propio. Esto hace que su traducción sea un auténtico desafío, incluso si se da entre lenguas cercanas. Cuando la equivalencia interlingüística es nula, la paráfrasis se convierte en la técnica traductológica más recurrida. En este estudio exploratorio, nos planteamos qué ocurriría si la lengua meta fuera la lengua de signos española, una lengua de carácter visogestual propia de la comunidad sorda. Mediante un estudio de caso realizado con 26 estudiantes del programa de Lengua de Signos Española y Comunidad Sorda, comprobamos que, efectivamente, la paráfrasis fue la fórmula más utilizada en las 76 traducciones analizadas de grabaciones en vídeo originadas durante el proceso de enseñanzaaprendizaje con los estudiantes; por tanto, es un procedimiento verbal que suele aplicarse en la traducción paremiológica ante la falta de correspondencia, independientemente de la modalidad lingüística de las lenguas implicadas. Se inicia así una línea de investigación que viene a generar nuevos interrogantes y a aportar nuevas perspectivas a la fraseología y la traductología.
\end{abstract}

Palabras clave: comunidad sorda; lengua de signos española; paremias; traducción; unidades paremiológicas.

\begin{abstract}
Paremia are part of the cultural heritage of each community and have their own idiomatic and figurative sense. This fact makes their translation a challenge, even if this takes place between related languages. When linguistic equivalence does not exist, paraphrasing is the translating technique most used. In this exploratory study, we take into consideration what would happen if the target language was Spanish Sign Language, a visual gestural language used by the Deaf community.
\end{abstract}

Recibido: 2021-05-28 / Aceptado: 2021-08-20 / Publicado: 2022-02-11

https://doi.org/10.17533/udea.ikala.v27n1a02

Editora: Doris Correa, Universidad de Antioquia, Medellín, Colombia

Derechos patrimoniales, Universidad de Antioquia, 2022. Este es un artículo en acceso abierto, distribuido según los términos de la licencia Creative Commons BY-NC-SA 4.0 Internacional. 
Using a case study conducted with 26 students enrolled in the Spanish Sign Language Program and among the Deaf Community we were able to demonstrate that paraphrasing was indeed the most commonly used technique and, thus, a verbal procedure that is usually applied for translating paremiological units when there is a lack of linguistic correspondence, regardless of the linguistic modality of the languages involved. Thus, a new line of research arises, raising new questions and bringing new perspectives to phraseology and translation studies.

Keywords: Deaf community; Spanish sign language; paremia; translation; paremiological units.

\section{RÉSUMÉ}

Les parémies font partie du patrimoine culturel de chaque peuple, avec leur propre signification idiomatique et métaphorique. Cela fait de leur traduction un véritable défi, même quand il s'agit de langues proches. Lorsqu'il n'y a pas d'équivalence interlinguistique, la paraphrase devient la technique de traduction la plus courante. Dans cette étude exploratoire, nous examinons ce qui se passerait si la langue cible était la langue des signes espagnole, une langue visuo-gestuelle spécifique à la communauté sourde. En utilisant une étude de cas realisée avec 26 étudiants du programme de Langue des Signes Espagnole et Communauté Sourde, nous avons démontré que, en effet, la paraphrase était la formule la plus utilisée et que, par conséquent, il s'agit d'un procédé verbal qui est habituellement appliqué en traduction parémiologique lorsqu'il y a un manque de correspondance, indépendamment de la modalité linguistique des langues impliquées. C'est le début d'une ligne de recherche qui va générer de nouvelles questions et apporter de nouvelles perspectives à la phraséologie et à la traductologie.

Mots-clés : traduction ; communauté sourde ; langue des signes espagnole ; parémie ; traduction ; unités parémiologiques.

\section{RESUMO}

As parémias fazem parte do património cultural de cada povo, com o seu próprio sentido idiomático e metafórico. Isto torna a sua tradução um verdadeiro desafio, mesmo que ocorra entre línguas próximas. Quando a equivalência interlinguística é nula, a parafraseação torna-se a técnica de tradução mais usada. Neste estudo exploratório consideramos o que aconteceria se a língua-alvo fosse a língua gestual espanhola, uma língua de caráter visuo-gestual típica da comunidade surda. Por meio de um estudo de caso efetuado com 26 estudantes do programa de Língua de Signos Espanhola e Comunidade Sorda, comprovamos que, de fato, a parafraseação foi a fórmula mais utilizada e, portanto, é um procedimento verbal que é normalmente aplicado na tradução paremiológica na ausência de correspondência, independentemente da modalidade linguística das línguas envolvidas. Assim começa uma linha de investigação que vem gerar novas questões e fornecer novas perspetivas para a fraseologia e tradução.

Palavras-chave: comunidade surda; língua gestual espanhola; parémias; tradução; unidades paremiológicas. 


\section{Introducción}

La traducción (e interpretación) de paremias entre lenguas, más o menos cercanas, suele plantearse como un reto de difícil -o imposible- solución, aludiendo a la carga cultural de aquellas y a su sentido idiomático y metafórico (Sevilla y Sevilla, 2005, párr. 1). En las últimas décadas, la traducción fraseológica ha sido objeto de reflexión teórica e investigación desde diferentes perspectivas (Timofeeva, 2012, p. 406). Sin embargo, no se encuentran referencias sobre la traducción de unidades paremiológicas entre lenguas de modalidad lingüística diferente, como es el caso de la lengua escrita española y la lengua de signos española (LSE).

En este artículo utilizamos la expresión "lengua de signos", porque es como tradicionalmente se ha empleado en España y como han sido reconocidas las lenguas de signos legalmente en este país, aunque coincidimos con Storch (1998, pp. 32-35) en que existen motivos lingüísticos y etimológicos suficientes para emplear el término "lengua de señas", como ocurre en los diferentes países de Hispanoamérica.

Factores históricos, culturales, lingüísticos y sociales han hecho de la LSE una lengua única y singular, de modalidad visual y gestual; una lengua minoritaria y minorizada que "reúne todas las características como lengua, cumple con las funciones que le son propias y es lengua de cultura" (Centro de Normalización Lingüística de la Lengua de Signos Española, 2014, p. 29). Una cultura propia y diferenciada, que se transmite mediante una lengua ágrafa y prácticamente carente de enunciados sentenciosos de origen anónimo y uso popular.

Nuestra intención, en este estudio exploratorio, es comprobar si en la LSE, al igual que ocurre con la equivalencia nula en las lenguas escritas, se recurre a la paráfrasis como principal técnica de traducción de paremias. Para ello, analizamos una actividad formativa introductoria, realizada por un grupo de estudiantes en traducción e interpretación signada, lo que refleja la importancia que le asignamos a la instrucción en traducción paremiológica durante su formación.

Aunque posiblemente, desde un punto de vista metodológico y para alcanzar un mayor rigor científico, hubiera sido deseable llevar a cabo este estudio con intérpretes profesionales -cuestión que esperamos abordar en un futuro próximo-, en este momento histórico, para la formación de traductores e intérpretes de LSE en España, con su reciente traslado al ámbito universitario, es prioritario prestar atención a su proceso de enseñanza-aprendizaje. En concreto, y al igual que ocurre con las lenguas orales, el entrenamiento en la traducción de unidades paremiológicas a la LSE durante el periodo formativo es esencial. Esto nos permitirá ofrecer a la comunidad sorda, y a la sociedad española en general, graduados universitarios con alta competencia lingüística y traductológica.

Para alcanzar nuestro objetivo, y determinar las principales técnicas de traducción empleadas por el alumnado, tomamos como referente la clasificación de Hurtado (2001, pp. 269-271):

- Adaptación: el traductor sustituye un elemento cultural por otro de la cultura receptora.

- Ampliación lingüística: se añaden elementos lingüísticos para alcanzar la equivalencia.

- Amplificación: el traductor precisa aspectos que no aparecen en el original.

- Calco: una palabra o sintagma se traduce literalmente.

- Compensación: una información determinada o un efecto estilístico se incluye en otro lugar distinto al original.

- Compresión lingüística: se resumen o condensan elementos lingüísticos.

- Creación discursiva: se realiza una equivalencia efímera, solo aceptable en dicho contexto.

- Descripción: se sustituye el término por la descripción de su forma o función. 
- Elisión: se eliminan u omiten elementos de información que aparecen en el original.

- Equivalente acuñado: se usa un término o expresión que está reconocida como equivalente, ya sea por el uso lingüístico o por el diccionario.

- Generalización: se usa un término más amplio, general o neutro.

- Modulación: hay un cambio de punto de vista, de enfoque o categoría.

- Particularización: al contrario que en la generalización, aquí el traductor opta por un término más preciso o concreto.

- Préstamo: se utiliza tal cual un término o expresión de la lengua origen.

- Sustitución (lingüística/paralingüística): cierta información lingüística se sustituye por otra paralingüística, como los gestos y la entonación, o al contrario. Se suele utilizar en la interpretación de lenguas.

- Traducción literal: se realiza la traducción, palabra por palabra, de un sintagma o expresión.

- Transposición: se cambia la categoría gramatical.

- Variación: se modifican elementos lingüísticos y paralingüísticos propios de una variedad lingüística por los de otra.

En este artículo, a continuación presentamos los rasgos distintivos de la comunidad sorda, un colectivo que tiene en la lengua de signos su principal valor cultural. Luego, nos adentramos en la traducción paremiológica y en las dificultades a las que se enfrentan los traductores y los formadores de futuros profesionales. Después, hacemos lo propio con la traducción signada, para la que argumentamos su existencia, describimos sus características distintivas e indicamos las carencias halladas sobre las paremias en estas lenguas. Seguidamente, y tras enunciar el enfoque metodológico, exponemos los resultados alcanzados en este trabajo y realizamos la discusión de estos. Por último, presentamos las principales conclusiones, entre las que destaca que, efectivamente, la paráfrasis es la técnica traductológica más utilizada cuando existe una equivalencia nula de las paremias en la lengua meta, pero que, sobre todo, esto es independiente de si esta lengua es oral, escrita o signada.

\section{Marco teórico}

Abordar el análisis de la traducción a LSE de elementos lingüísticos con un peso cultural tan importante como son las paremias requiere, sin lugar a duda, de una aproximación previa a la naturaleza y las particularidades de la comunidad sorda y de su lengua. En esta sección describimos las principales características culturales y lingüísticas de este colectivo, para, seguidamente, dar cuenta de la tipología de paremias que existe en español y de las trabas que genera su traslación a una segunda lengua escrita. También se precisan los conocimientos y las competencias que, según diferentes autores, son indispensables que adquieran los estudiantes de traducción para hacer frente al reto de traducir paremias. Por último, se justifica el uso del término "traducción" cuando una de las lenguas implicadas es de modalidad viso-gestual y ágrafa, y reparamos en la falta de referencias sobre las unidades fraseológicas en las lenguas signadas.

\section{Claves para entender a la comunidad y a la cultura sordas}

La imagen más habitual de la sordera es aquella centrada exclusivamente en la pérdida auditiva, en las dificultades para oír de estas personas y en las limitaciones que tienen para la adquisición y el desarrollo del lenguaje oral. Esta perspectiva médica, tan arraigada en nuestra sociedad y que entiende la sordera como una patología, ha generado diversas respuestas (re)habilitadoras para conseguir su plena integración social mediante el desarrollo de la audición, el habla, la lectura labial y el uso de prótesis auditivas.

Frente a esta visión dominante, se ha generado un enfoque de la sordera diametralmente opuesto. La perspectiva sociocultural, en la que la sordera se valora positivamente, surge en Estados Unidos en la década de los sesenta del siglo pasado a raíz 
de los trabajos del lingüista William Stokoe, que reconocían la lengua de signos americana como una lengua verdadera (cfr. Herrero, 2009, p. 12; Rodríguez, 1992, p. 21). Este autor hizo que las diferentes lenguas de signos del mundo se convirtieran en objeto de estudio, pero, sobre todo, se originó un cambio de percepción en el propio colectivo de personas sordas: pasaron a verse como miembros de una comunidad, con una historia compartida, unas costumbres propias y una forma diferente de entender el mundo que les rodea.

A deaf community is a group of people who live in a particular location, share the common goals of its members, and in various ways, work toward achieving these goals. A deaf community may include persons who are not themselves Deaf, but who actively support the goals of the community and work with Deaf people to achieve them (Padden, 1980, p. 92).

Esta clásica definición subraya el uso diferenciado entre sordo y Sordo, aunque autores como Kusters et al. (2017, pp. 13-15) sostienen que, dada la complejidad propia de la sordera en cuanto a identidades y prácticas lingüísticas, la distinción "s/S" supone una mera simplificación y apuestan por el uso del término "sordo" para individuos, entidades o conceptos teóricos - propuesta que empleamos en este artículo-. "This uppercase Deaf and lowercase deaf terminology reflects fundamentally different ways of coping with and feeling about hearing loss" (Leigh y Andrews, 2017, p.XIII). Así, mientras que la minúscula se emplea con aquellas personas con pérdida auditiva que utilizan la lengua oral a diario y que no se consideran miembros de una minoría lingüística, la mayúscula designa a aquellas personas que se han revelado contra el enfoque médico imperante, que se sienten orgullosos de ser sordos, de ser parte de una cultura compartida y de utilizar una lengua signada tan rica y compleja como las orales.

La forma en la que entendemos y concretizamos el mundo está íntimamente relacionada con nuestra lengua y, por eso, "los sordos poseen una cultura distinta producto de una interacción particular y del hecho de percibir el mundo de una manera especial. Los sordos se caracterizan, pues, por poseer una cultura eminentemente visual" (Massone et al., 2003, pp. 22-23). Según Padden (1980, pp. 95-98), uno de los principales valores de la cultura sorda es su historia y literatura, cuya transmisión intergeneracional se realiza mediante la tradición signada. A esto se unen sus creencias y comportamientos, construidos con base en su condición de personas visuales y en la opresión de una mayoría oyente, y, sobre todo, considera que la lengua de signos es su principal bien cultural, porque les identifica, define y cohesiona como grupo.

\section{Las lenguas de signos como lenguas naturales de las personas sordas}

Las lenguas de signos son lenguas minoritarias, a tenor del número de usuarios: tan solo unas 13000 personas, mayores de 6 años, en España, la utilizan como principal medio de comunicación, según los últimos datos oficiales (Instituto Nacional de Estadística, 2008). Pero, además, por su situación frente a las lenguas de modalidad auditivo-oral, son lenguas históricamente minorizadas, "by institutions, policies and research which ignore or even explicitly exclude them" (De Meulder y Murray, 2017, p. 137).

En las últimas décadas, entidades representativas de la comunidad sorda en todo el mundo han luchado por el reconocimiento legal de estas lenguas y, como consecuencia, de sus derechos lingüísticos. En el caso de España, la publicación de la Ley 27/2007, del 23 de octubre, supuso el reconocimiento explícito de la lengua de signos española y de la catalana, y del derecho a su aprendizaje, conocimiento y uso. Sin embargo, como señalan Esteban y Ramallo (2019, p. 36), esta norma se elaboró desde el enfoque de la discapacidad, mermando así la perspectiva de los derechos lingüísticos. Aunque es una herramienta jurídica fundamental para mejorar la calidad de vida de la comunidad sorda, hoy en día sigue sin estar plenamente desarrollada y las expectativas que generó no llegan a cumplirse (cfr. Cabeza-Pereiro y Ramallo, 2016, pp. 11-16). 
Esta ley define las lenguas de signos como "sistemas lingüísticos de carácter visual, espacial, gestual y manual en cuya conformación intervienen factores históricos, culturales, lingüísticos y sociales" (art. 4). Entre estos factores, queremos destacar: la heterogeneidad de la comunidad sorda y el escaso número de signantes nativos - solo el $5 \%$ tiene progenitores sordos-; la importancia, por tanto, de una transmisión entre individuos de la misma generación — porque, además, son lenguas ágrafas-; la discriminación, e incluso prohibición, que se da en los contextos educativos para su aprendizaje y uso; la situación de diglosia que se da en nuestras sociedades, etc. (Costello et al., 2012, p. 371; De Meulder y Murray, 2017, p. 148).

Como también se señala en dicha definición, las lenguas de signos se caracterizan por ser lenguas de modalidad viso-gestual, que se perciben visualmente y se transmiten gestual y manualmente. Además, explotan capacidades expresivas, como son el uso del espacio, la iconicidad, la expresión facial y corporal, o la simultaneidad de los articuladores, y que, en muchos casos, tienen valor gramatical. Las palabras de Herrero (2009) nos permiten ilustrar las particularidades de estas lenguas:

[...] el uso que la narración signada hace del espacio, sobre todo cuando se accede al código gestual, con continuos movimientos del cuerpo y flexionando y adaptando sus manos a la representación como los fonemas de las lenguas orales no podrían hacer jamás; la posibilidad de mantener dos referencias simultáneas, cada una en una mano; o la de representar al otro en un estilo directo muchísimo más frecuente que en nuestras lenguas orales, pues afecta a personas, a animales, incluso a cosas (p. 13).

A esto se une una serie de procedimientos gramaticales poco comunes en las lenguas orales indoeuropeas y que, al ser expresados, además, manualmente, hacen que desde nuestra perspectiva fonocéntrica no los reconozcamos como propios de una lengua, tales como la clasificación, la incorporación o la concordancia universal del predicado con todos los argumentos (Herrero, 2009, p. 11).

\section{La traducción paremiológica}

Según Corpas, "la 'Paremiología' no es más que el estudio de una parte de la Fraseología” (Corpas y Ortiz, 2017, p. 276). Esa parte son las paremias, archilexema que se define como

$$
\begin{aligned}
& {[\ldots] \text { una unidad fraseológica (UF) constituida por un }} \\
& \text { enunciado breve y sentencioso, que corresponde a una } \\
& \text { oración simple o compuesta, que se ha fijado en el } \\
& \text { habla y que forma parte del acervo socio-cultural [sic] } \\
& \text { de una comunidad hablante (Sevilla y Crida, 2013, } \\
& \text { p. 106). }
\end{aligned}
$$

Estos autores clasifican las paremias españolas en seis categorías: por un lado, proverbios y aforismos (de origen conocido y uso preferentemente culto) y, por el otro, refranes, frases proverbiales, locuciones proverbiales y dialogismos (de origen anónimo y uso preferentemente popular). Nuestro interés se centra en este último grupo y, más concretamente, en los refranes y en las frases y locuciones proverbiales.

\section{El refrán es}

[...] una paremia de origen anónimo y uso popular, cuya estructura es generalmente bimembre, con presencia de elementos mnemotécnicos, con potencial presencia de elementos jocosos, basado en la experiencia y con valor de verdad universal, en su gran mayoría (Sevilla y Crida, 2013, p. 111),

y es la más numerosa de este tipo.

La frase proverbial, en cambio, es unimembre, no presenta elementos mnemotécnicos, puede incluir elementos jocosos, tiene valor de verdad universal y se basa en la experiencia.

Por último, la locución proverbial posee una estructura oracional, con un núcleo verbal conjugable, aunque coincide con la frase proverbial en el hecho de presentar valor de verdad absoluta y un origen experiencial, así como en la posibilidad de que se incluyan elementos jocosos (Sevilla y Crida, 2013, p. 112).

Las paremias se han convertido en una de las principales preocupaciones en la teoría y la práctica de la traducción (Richart, 2008, p. 1). Según Cobeta 
(2000), la complejidad propia del proceso traductológico se incrementa cuando el traductor (o el intérprete) se enfrenta a las paremias: su peculiaridad y especificidad hacen que la traducción paremiológica se considere uno de los grandes retos para todo traductor. Entre estas dificultades están la idiomaticidad, las estructuras sintácticas atípicas, la confluencia de diferentes registros o su uso en ámbitos científicos variados (cfr. Cobeta, 2000, p. 266). Incluso se ha planteado la intraducibilidad de algunas unidades fraseológicas (UF), aludiendo a las referencias históricas o socioculturales que contienen (Timofeeva, 2012, p. 407). No obstante, lo que sí parece claro es que presentan, en palabras de Richart (2008), cierta resistencia a la traducción, debido a su falta de transparencia, lo que obliga "al traductor a realizar un acto de reescritura que sustancialmente no es distinto de la escritura misma" (p.6).

Este acto de reescritura representa las técnicas y estrategias con las que el traductor trata de alcanzar la ansiada equivalencia. Según Corpas (2003), el proceso de traducción de UF requiere, en primer lugar, de su identificación e interpretación en el (con)texto dado, para, luego, buscar las correspondencias "primero en el nivel lexicológico, el plano teórico, para, a continuación, bajar a los niveles textual y discursivo, en una palabra, al plano real" (p. 216). Timofeeva (2012, p. 411) indica que, en el primer nivel léxico, se busca lograr una equivalencia semántica de la UF original, equivalencia que, en opinión de Corpas (2003, pp. 217-219), existe en tres niveles: total, parcial y nula (en esta última no existe correspondencia en la lengua meta y el traductor debe utilizar la paráfrasis u otras técnicas que impiden mantener los valores connotativos). En cuanto al plano textual, que completa el establecimiento de las equivalencias, Corpas (2003, p. 219) distingue tres dificultades para el traductor: no identificar la UF como tal, lo que indica una falta de competencia lingüística (Timofeeva, 2012, p. 412); que la identifique, pero que no sepa interpretarla para ese contexto, lo que supondría un problema de competencia comunicativa (Timofeeva, 2012, p. 412); y, por último, saber reconocerla e interpretarla, pero no ofrecer una equivalencia adecuada en la lengua meta, lo que representa una competencia traductológica limitada (Timofeeva, 2012, p. 413).

\section{La formación en traducción paremiológica}

Llegar a ser un traductor profesional requiere de una formación específica que permita la adquisición de la competencia traductora, compuesta por seis subcompetencias: bilingüe, extralingüística, conocimientos de traducción, profesional o instrumental, psicofisiológica y estratégica (PACTE, 2003, pp. 16-17). La subcompetencia bilingüe supone tener conocimientos procedimentales para la comunicación en ambos idiomas — pragmáticos, sociolingüísticos, textuales, gramaticales y léxicos-, incluyendo el control de interferencias cuando se alterna entre ellos.

Para Corpas (2013, p. 337), en el nivel más alto de la competencia de los hablantes de una lengua, sea materna, segunda o extranjera, se encuentra la competencia fraseológica: reconocer, entender y utilizar — reverbalizar, en el caso de traductores e intérpretes (Corpas y Ortiz, 2017, p. 274) - adecuadamente las uf en el contexto. Así se refleja en el Marco común europeo de referencia para las lenguas (MCER), concretamente en la competencia lingüística (léxica) y en la sociolingüística, aunque

\footnotetext{
No se proporciona información sobre qué tipos de unidades se debe incluir en cada uno de los niveles que reconoce el MCER, ni con qué profundidad se debe tratar una determinada unidad en cada uno de los niveles, etc. (Corpas, 2013, pp. 367-368).
}

Esta competencia fraseológica es esencial para la traducción, aspecto preocupante en los estudiantes universitarios de Traducción e Interpretación. Martín (2008, p. 195) encontró que su grado de conocimiento de paremias y fórmulas del español es insuficiente, tanto en la identificación como en su interpretación. También observó la importancia de tener este conocimiento para realizar transferencias a la lengua extranjera. Este autor 
concluye que es esencial que el alumnado reciba una formación teórica y práctica sobre UF del español, lo que facilitará el desarrollo de la inferencia metafórica en esta lengua y en la extranjera (además de enseñar las uf de esta).

Un ejemplo de aplicación didáctica de la traducción paremiológica lo encontramos en Díaz y Sabio (2017, pp. 116-125), quienes diseñan una serie de actividades para desarrollar en el alumnado las subcompetencias traductológicas, mientras recorren las fases de la traducción de paremias, tomando conciencia de las dificultades y de las posibles técnicas y estrategias.

\section{La traducción de paremias a la lengua de signos española}

En los últimos años, la traducción y la interpretación han dejado de verse exclusivamente desde una perspectiva fonografocéntrica, en la que el carácter hablado y escrito de los textos diferenciaba estas actividades. De acuerdo con Pöchhacker (2004, p. 10), la distinción está en la inmediatez del proceso y no en la modalidad de las lenguas implicadas. Además, las nuevas tecnologías han hecho posible que la permanencia de un texto no solo sea escrita. Así, es cada vez más frecuente la traducción de lenguas signadas, desde un formato escrito a uno videográfico, y viceversa (sin descartar otras opciones).

En opinión de Segala (2010, p. 28), la traducción signada es un tipo de traducción interlingüística, intersemiótica e intermodal, en la que se involucra una lengua auditivo-oral y una kinésico-visualespacial. No obstante, en ocasiones se puede dar una traducción interlingüística, sin que sea intersemiótica ni intermodal, cuando ambas lenguas son signadas y el soporte de los textos es en vídeo. Quadros y Segala (2015, pp. 360-363) argumentan que esta traducción intermodal presenta características propias, como un solapamiento de elementos de la traducción y de la interpretación, y la visibilidad necesaria e identidad explícita del traductor (que, preferiblemente, suele ser un profesional sordo).
En cuanto a las técnicas de traducción signada, Quadros y Souza (2008, pp. 181-86) localizan las siguientes: la explicitación (por medio de la aclaración del texto de origen en la lengua meta e incluyendo las ejemplificaciones que suele realizar motu proprio el traductor), la transliteración o préstamo lingüístico de la lengua oral (mediante el deletreo de términos, utilizando el alfabeto dactilológico), el préstamo lingüístico desde otras lenguas de signos, la creación de signos ante neologismos, etc.

Por otro lado, el número de publicaciones sobre la traducción de la LSE es anecdótico, entre las que destaca el trabajo de González-Montesino y Saavedra-Rodríguez (2020). En él analizan la traducción de las metáforas del vino a la LSE, aplicando las técnicas propuestas por Hurtado (2001, pp. 269-271), y encuentran que la amplificación (o paráfrasis) fue la principal técnica empleada, seguida de la descripción.

En nuestra búsqueda bibliográfica para este estudio no hemos hallado referencia alguna sobre la traducción de paremias a la LSE o a otras lenguas signadas. No obstante, lo más llamativo es que existe un importante vacío en el registro e investigación de las UF en LSE: ni hay publicaciones científicas, ni diccionarios o repertorios monolingües/bilingües específicos sobre las paremias. Esta situación es entendible si tenemos en cuenta que la primera descripción lingüística de esta lengua fue en 1992, con la publicación del trabajo de Rodríguez titulado Lenguaje de signos.

En todo caso, después de una intensa búsqueda sobre paremias de origen anónimo y uso popular en LSE, y más allá de algunos vídeos elaborados por personas sordas en Internet, solo podemos referenciar los números 3 y 4 de la revista As nosas mans (Asociación de Personas Sordas de Santiago de Compostela, 2008). Estos incluyen algunas locuciones del gallego, su explicación en español y el equivalente en la LSE, que es generalmente un solo signo léxico (como, por ejemplo, las expresiones 'tan bo e Xan coma Pericán' y 'non ter papas na boca’ que aparecen en la página 22 del número 3 ). 
Lo que sí hemos comprobado es que en estas lenguas también se da esa falta de concreción de las UF en los distintos niveles de competencia de la que habla Corpas (2013, p. 340). Tomando como referente el proyecto Signed languages for professional purposes (Leeson et al., 2016, pp. 6-8), que especifica los niveles de competencia según los descriptores del MCER para las lenguas de signos en la formación superior (especialmente de intérpretes), descubrimos que se hace mención explícita al dominio de las "expresiones idiomáticas" en el nivel $\mathrm{C} 1$ de la competencia general de comprensión (p. 21) y en el C2 para la competencia general en interacción signada (p. 29). También se hace alusión expresa en el nivel C2 de la competencia sociolingüística, mientras que en el $\mathrm{C} 1$ se señala la capacidad para reconocer una amplia gama de expresiones idiomáticas (p. 38). Por último, se indica la necesidad de contar con un vasto repertorio léxico, que incluya dichas expresiones en el nivel C2 de competencia léxica (p. 40).

Lógicamente, estos estándares se deben tener en cuenta en la formación de traductores e intérpretes de LSE. Sin embargo, a diferencia de lo que ocurre en las lenguas orales, no existen materiales para la enseñanza y el aprendizaje de la LSE adaptados al nivel C1 y C2 del MCER. A esto se une que la formación a nivel universitario es muy reciente: en el curso 2016/2017 comenzó la implantación progresiva del Grado en Lengua de Signos Española y Comunidad Sorda en la Universidad Rey Juan Carlos, único título en nuestro país que aborda la enseñanza de esta lengua con fines profesionales.

Por todo lo expuesto en este apartado, podemos afirmar que la traducción paremiológica a la LSE y su didáctica se presentan como auténticos desafíos.

\section{Método}

Ante la falta de publicaciones respecto de la traducción de unidades paremiológicas a lenguas de signos, este estudio adopta un método de tipo cualitativo y el estudio de caso como metodología de investigación, ya que permite hacer frente a la complejidad que implica el análisis del proceso de traducción (Neunzig y Tanqueiro, 2007, p. 37). La técnica de investigación utilizada fue la observación del material videográfico recopilado para este estudio, formato esencial, porque nos ofrece un registro objetivo del caso, que facilita su posterior análisis (Simons, 2011, p. 94).

La población objeto de estudio es el alumnado de la asignatura "Aplicación de las técnicas de interpretación a la lengua de signos española IV" del Grado en Lengua de Signos Española y Comunidad Sorda de la Universidad Rey Juan Carlos (curso 2019/20). La muestra corresponde al grupo de la mañana, seleccionada por conveniencia, al ser plenamente accesible por nuestra condición de docentes. Esta clase está compuesta por 38 estudiantes, aunque solo 34 optaron por evaluación continua; solo hay 3 varones, entre ellos una persona sorda, cuya lengua materna es la LSE, y 3 de las alumnas ya son intérpretes profesionales.

Se declara que, para la realización del presente estudio, se han seguido las normas éticas de investigación, en cuanto a la recolección y el tratamiento de los datos, y que se obtuvo consentimiento informado de todos participantes para emplear las grabaciones en vídeo y en audio originadas durante el proceso de enseñanza-aprendizaje en actividades de carácter investigador - de acuerdo con lo dispuesto en el Reglamento UE 679/2016, del 27 de abril, sobre la protección y el tratamiento de datos personales, del Parlamento Europeo y el Consejo de la Unión Europea, así como en la Ley Orgánica 3/2018 de España, del 5 de diciembre, de protección de datos de carácter personal y garantía de los derechos digitales-. Además, se han seguido las recomendaciones o directrices del Comité de Ética de la Investigación de la Universidad Rey Juan Carlos para la realización de proyectos de investigación con participación de alumnado de esta universidad.

El material videográfico utilizado como corpus es el resultado de una actividad complementaria de 
aprendizaje, no obligatoria y no evaluable, desarrollada a distancia del 22 al 29 de abril de 2020, por el confinamiento decretado por la COvid- 19. Se planteó como foro en el aula virtual, para permitir que los estudiantes pudieran consultar las traducciones del resto de sus compañeros, y fomentar de este modo el intercambio de ideas y conocimientos. Las instrucciones fueron:

1. Cada estudiante tenía que elegir tres refranes o dichos de los incluidos en Jordán (2010, pp. 324-331).

2. Después de informar de su elección en el foro, para evitar la duplicación y abarcar el mayor número de paremias, debían consultar las fuentes necesarias para realizar la traducción a LSE.

3. Una vez decididos los equivalentes, grabarlos en vídeo por separado e incrustarlos en el foro.

4. En la última clase online síncrona, se comentarían las principales dificultades y algunas de las traducciones propuestas.

Se trata, por tanto, de una actividad formativa introductoria similar a una de las propuestas por Díaz y Sabio (2017, pp. 116-117), centrada en la búsqueda de correspondencias a nivel léxico como paso previo a la enseñanza en el plano textual y discursivo. Por este motivo, no entraremos a valorar la adecuación o no de las correspondencias planteadas. En la actividad participaron 26 de los 34 estudiantes posibles $(\approx 76 \%)$ y de las 78 traducciones hemos eliminado 2 , porque las paremias no estaban incluidas en Jordán (2010).

Por otro lado, queremos advertir que la compilación de vídeos es ilustrativa y no presenta la uniformidad y exhaustividad requeridas para un estudio de corpus. El procedimiento de investigación fue: examinar cada uno de los vídeos, transcribir a glosas (véase Anexo) las traducciones en LSE mediante una adaptación del sistema de glosas propuesto por Herrero (2009) y, por último, clasificar cada traducción según las técnicas utilizadas.

En el siguiente apartado se exponen los resultados y, para facilitar su lectura, hemos decidido incluir la discusión. También añadimos fotogramas de algunos vídeos como ejemplos y, aunque el alumnado firmó al inicio del curso un consentimiento informado para que las grabaciones generadas en el proceso de enseñanza-aprendizaje pudieran ser objeto de estudio, hemos pixelado los rostros para mantener su anonimato y evitar los problemas éticos de permisos y de derechos de autor que supone investigar a través de vídeos (Flick, 2015, p. 130). Aun así, hemos consultado a las tres estudiantes que aparecen en las imágenes su conformidad de que se publiquen de forma pixelada — como se muestran en las Figuras 2 y 3 -, y se ha obtenido, además, un consentimiento informado específico como participantes del estudio.

\section{Resultados y discusión}

Los resultados alcanzados en este estudio demuestran una preponderancia en el uso de la técnica de amplificación a la hora de traducir paremias a la LSE. En esta sección se muestra hasta qué punto y de qué forma este procedimiento traductológico es el principal recurso utilizado por los estudiantes cuando se enfrentan al reto de trasladar dichas unidades a una lengua signada. Además, se describe el modo en el que han sido empleadas otras técnicas que podríamos calificar como excepcionales.

\section{La amplificación como técnica preferente y su tipología de uso}

En la Figura 1 podemos ver que, en las 76 traducciones, el alumnado aplicó la técnica de amplificación en 64 ocasiones (84,2\%). Para el resto utilizaron la técnica de equivalente acuñado, 10 veces $(13,1 \%)$, y solo 1 vez la de descripción $(1,3 \%)$ y la de calco $(1,3 \%)$. Las 14 técnicas restantes propuestas por Hurtado (2001) no fueron empleadas en este estudio.

Por lo tanto, en la traducción de refranes y dichos del español a la LSE, encontramos que la aplicación de la amplificación es significativamente mayor que la del resto de técnicas. Esta técnica supone la inclusión, en el texto meta, de "precisiones no 
Figura 1 Técnicas aplicadas en la traducción paremiológica a la LSE

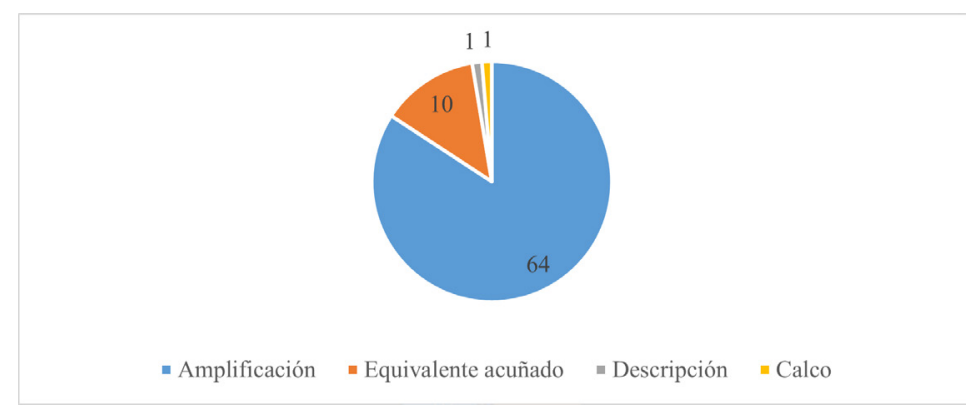

formuladas en el texto original: informaciones, paráfrasis explicativas, notas del traductor, etc." (Hurtado, 2001, p. 269). Aunque nuestro análisis es a nivel léxico, el resultado coincide con lo que ocurre en la traducción escrita de UF cuando hay equivalencia nula entre las lenguas: el uso preferente de la paráfrasis (Corpas, 2003, pp. 217-218). Parece, pues, que la modalidad lingüística del par de lenguas implicadas no influye en el tipo de procedimiento verbal aplicado por el traductor ante paremias sin equivalente en la lengua meta.

Este resultado también concuerda con el uso preferente de la amplificación en la traducción de las metáforas del vino a la LSE (GonzálezMontesino y Saavedra-Rodríguez, 2020) y con una de las técnicas detectadas por Quadros y Souza (2008, pp. 181-182), aunque la denominan "explicitación". Estos autores incluyen aquí las ejemplificaciones.

Según Rodríguez (1992), la expresión de la condicionalidad (real e hipotética) en la LSE utiliza de forma explícita el signo EJEMPLO en la prótasis, acompañado de la expresión facial característica, lo que pone de relieve "el hecho de ser enunciados basados en hábitos de observación creados por la experiencia” (p. 317). Recordemos que esta característica experiencial la presentan los refranes, las frases y las locuciones proverbiales del español (Sevilla y Crida, 2013, pp. 111-112). Por su parte, Herrero (2009) también afirma que "el signo EJEMPLO se emplea para todo tipo de condicionales (reales, irreales, probables), que en español se diferencian por los tiempos y modos verbales de las dos oraciones" (p. 337). Señala que el nexo EJEMPLO se suele aislar de la oración a la que se aplica y se articula con la expresión facial que comentaba Rodríguez (1992, pp. 250-251): una entonación ciliar ascendente. Dicha entonación es la única marca de la composición cuando se omite el nexo, algo que suele darse en el discurso espontáneo (Herrero, 2009, p. 337).

En nuestro corpus hemos localizado hasta 27 estructuras condicionales como solución para traducir las paremias a la LSE, solo 4 de ellas utilizando el nexo EJEMPLO. Siguiendo a Quadros y Souza (2008, pp. 181-182), las hemos incluido en la categoría de "amplificaciones", ya que estas oraciones permiten precisar y aportar información no incluida en la paremia original, para facilitar su comprensión a las personas sordas.

A continuación presentamos los ejemplos de amplificación detectados: en la Tabla 1 solo aparecen las paráfrasis explicativas (41), mientras que en la Tabla 2 se incluyen los ejemplos de amplificación a través de paráfrasis en los que se utilizaron estructuras condicionales (destacadas en gris las que emplean el nexo EJEMPLO). En el Anexo pueden verse las glosas o convenciones.

\section{Técnicas traductológicas secundarias}

Tras haber descrito la que, con diferencia, fue la principal técnica empleada por el alumnado para traducir paremias del español a la LSE — la amplificación-, a continuación se muestra y analiza el conjunto de técnicas que podríamos calificar 
Tabla 1 Ejemplos del uso de la paráfrasis en la traducción de paremias a LSE

1. A perro flaco todo son pulgas

2. A quien madruga, Dios le ayuda

3. Cada barco que aguante su vela

4. Cada persona es un mundo

5. Comes más que una lima

6. De visita todos somos buenos

7. Del dicho al hecho

8. El perro ladrador no es mordedor

9. El saber no ocupa lugar

10. Eres más falso que Judas

11. Eres más largo que un día sin pan

12. Esto es coser y cantar

13. Haced lo que yo diga, pero no lo que yo haga

14. Haz bien sin saber a quien

15. La cara es el espejo del alma

16. La procesión va por dentro

17. La vida hoy está así

42
PERSONA ++ DÉBIL SIEMPRE MALA-SUERTE

TÚ RESPONSABLE DEBER / OBLIGACIÓN TUYA CUMPLIR DEBER

CADA-UNO HACER-LO-SUYO

PERSONA ++ TODAS IGUALret nO / PERSONA ++ CADA-UNA DIFERENTE ++

TÚ COMER ++

PERSONA CL:DOS-PERSONAS-SE-ENCUENTRAN TIEMPO EN-Un-MOMENTOt NO-PASA-NADA / PERO CL:DOSPERSONAS-SE-ENCUENTRAN PASAR-MUCHO-TIEMPO-DE-RELOJt AL-FINAL CHOCAR / PESADO

$\underline{\text { DECIR }++ \text { EN-REALIDADref PARA-NADA }}$

PERSONA DECIR++ ADVERTIR / ACCIÓN ADVERTENCIA CUMPLIRR TI NUNCA

cosa ++ saber ampliar-Conocimiento bloqueo ++ no-Ser / motivo saber sobrar nunca

TÚ MENTIR ++ MUCHO

ACCIÓN LARGO 0 PERSONA ALTAT / EXAGERADO

SITUACIÓN Fécll++"muY"

COSAS YO DECIRT COPIAR PODER / YO COSAS HACERT COPIAR NUNCA

PROTAGONISMO + + $\underline{\text { NO-HACER-FALTANEg }}$

IU CARA EXPRESIÓNT / EMOCIÓN SENTIMIENTOS EXPRESAR

dolor tristeza enfado dentro-cuerpo / contar no // esa persona contenta / "pero" en-

REALIDADret DENTRO-CUERPO ENFADO TRISTEZA DOLOR

AHORA ÉPOCA ES-ASI / FUTUROret INCÓGNITA

COSAS MAL ++ MUCHO ++

algo claro "clarísimo"

Yo SOLo[loq izq] / YO PERSONA $++[\operatorname{loq}$ dcha] Mala JUNTO / eso[loq izq] MEJOR

tú decir mentira[loq izq] / hablar[loq dcha] / eso[loq izq] más

DECIR ++ / EN-REALIDADret PARA-NADA

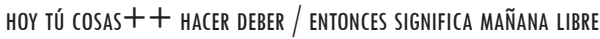

IEMA NORMAS GUSTOS DECISIONES PERSONA++ TODAS OPINIÓN SU PENSAR-COMÚNt / DIFíCIL / CASI IMPOSIBLE

hOY COSA ++ haber ++ poder / MaÑANa NO-SABERneg

PERSONA ++ MALA CONret noneg / YO SOLO PREFERIRafirm

YO PERSONA Cl:SEGUIR-A-UNA-PERSONA COPIAR + + QUIÉNret / PERSONA INTERESAR MÁS A-MÍ // DEPENDE PERSONA CADA-UNO DAR-me QUÉret/ Yo VALORAR INTERESAR Sí-0-NO

YO FALLO UNA-VEZI / ETIQUETAR-me PARA-SIEMPRE

A-TI ENGANART / NO

TÚ INTENTAR + + / PERO CONSEGUIR NONEg

Si tú PerSONa ++ madura no-habercond / CONtacto poder consecuencia negativa

YO A-TI VER-te $\mathrm{f} /$ TÚ CAMBIAR

PERSONA ++ TODAST / MANDAR QUERER

SI TÚ CRITICAR A-Mícond / AL-FINAL YO RESPONDER-te

SI TÚ DEL-RESTO RÉrcond / AL-FINAL DEVOLVER FASTIDIAR-me

TÚ SIEMPRE APROVECHAR / TÚ MUCHA-CARA 
Tabla 1 Ejemplos del uso de la paráfrasis en la traducción de paremias a LSE (Cont.)

37. Unos nacen con estrellas y otros estrellaos

38. Vas para detrás como los cangrejos

39. Vete a freír espárragos

40. Viene como anillo al dedo

41. Yo callo y ando persona ++ algunas suerte[loq dcha] nacer toca / persona ++ otras suerte[loq izq] Noneg

aVANZAR DESARROLLART Noneg / EN-REALIDAD IR-HACIA-ATrás aguantar no "no te aguanto"

SITUACIÓN ESTAt / Yo IMAGINaCIÓN / SE-RELACIONAN PERFEcto

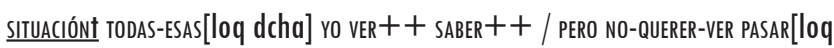
dcha] / Importante VIDa Continuar-adelante[loq izq] Problema SIN / PaSar[loq dcha]

Tabla 2 Ejemplos del uso de paráfrasis explicativas empleando estructuras condicionales en la traducción de paremias a LSE

\begin{tabular}{|c|c|}
\hline 42. A caballo regalado no le mires el diente & aYudar-me cond / Yo ACEPTAR / NADA-Más \\
\hline 43. A lo hecho, pecho & SI HACER YA HACERCONd / CONSECUENCIA ACEPTAR NO-QUEDA-OTRA \\
\hline 44. A mal tiempo, buena cara & SI SIIUACIÓN MAL TOCACOnd / TÚ ACTITUD POSITIVA \\
\hline 45. A palabras necias, oídos sordos & $\begin{array}{l}\text { SIUUACIÓN MI TÚ COSAS DECIR++ RELACIONADO-CONMIGO No-HABERCond / INTERESAR Noneg / } \\
\text { IGNorar-te }\end{array}$ \\
\hline $\begin{array}{l}\text { 46. Arrieros somos y nos encontraremos en el } \\
\text { camino }\end{array}$ & SI AYUDAR-me Nocond / FUTURO AYUDARTe ABURRIR No"no me apetece" \\
\hline 47. Cada oveja con su pareja & 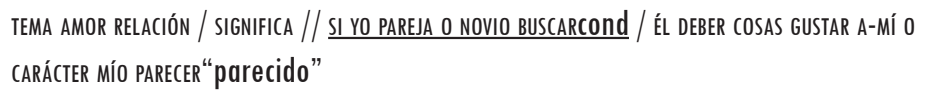 \\
\hline 48. Cuando el río suena, agua o piedras lleva & SI TÚ ALGO RARO VER HUELE-RAROCONd / SIGNFFICA ALGO TOCA SEGURO \\
\hline 49. De bien nacido es ser agradecido & SI PERSONA GRACIAS DECIRCONd / SIGNIFICA BUENO \\
\hline 50. De noche todos los gatos son pardos & SI PERSONA ALGO NEGATIVO O ALGO POSITIVO DAR-Me QUERERCONd / YO DESCONFIAR / DARSE-CUENTA DIF́́CIL \\
\hline 51. Dime con quién te juntas y te diré quién eres & PERSONA ++ Tú CONTACTO + + HABERCOnd / AL-FINAL COSTUMBre SUYA ADQUIRIR \\
\hline 52. Donde manda patrón, no manda marinero & SI PERSONA JEFE MANDAR-me cond / Yo AGACHAR-LAS-OREAS \\
\hline 53. Donde no hay cabeza tiene que haber pies & PERSONA OLVIDAR + + cond / CAMINAR DOBLE IDA-Y-VUELTA \\
\hline 54. El que calla otorga & PERSONA DECIR NADA SILENCIO MANOS-EN-BOLSILLOSCONd / IIGNFFICA RESPONDER-me DECIR YA \\
\hline 55. El que la líe que la deslíe & 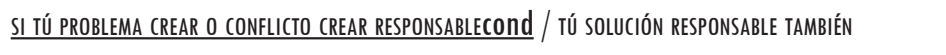 \\
\hline 56. El que se pica ajos come & PERSONA MOLESTAR-Se cond / ELt ALGO HACER"hecho" \\
\hline 57. Es peor el remedio que & 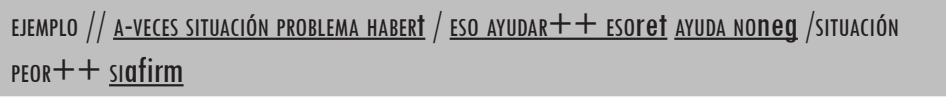 \\
\hline 58. Juan Palomo, yo me guiso y yo me lo como & SI Tú INTENTARCond / AL-FINAL CONSEGUR // SI IÚ INTENTAR NOCONd / TÚ CONSEGUIR NUNCA \\
\hline 59. Las cosas de palacio van despacio & COSAS IMPORTANTE HACER BIENCONd / TIEMPO DURAR HACER-FALTA \\
\hline 60. Lo que no vale se tira & SI ALGO NO-SERVIRCONd / MEJOR TIRAR \\
\hline 61. No tires la piedra y escondas la mano & 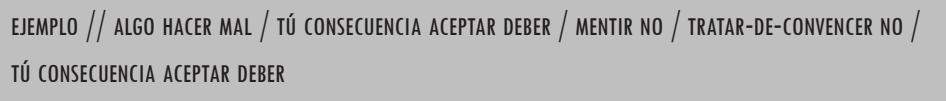 \\
\hline 62. No vendas la piel del oso antes de cazarlo & 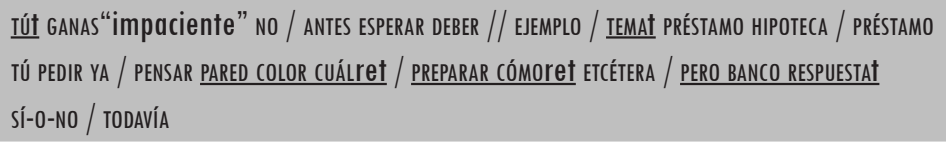 \\
\hline 63. Preguntando se va a Roma & SI DUDARCond / PREGUNTARafirm \\
\hline 64. Todo lo que nace muere & 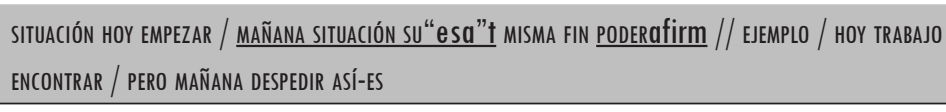 \\
\hline
\end{tabular}


Tabla 3 Ejemplos del equivalente acuñado en la traducción de paremias a LSE

\begin{tabular}{ll}
\hline 65. Con la música a otra parte & mudarse \\
66. Estás con el agua al cuello & LLENAR-te / AHOGADO \\
67. Estás más quieto que una estatua & tú figura \\
68. Esto me huele mal & esta situación apestar \\
69. Eres un sol & Tút Bueno \\
70. Eres un tarugo & tú tonto \\
71. Eres una cotorra & Tú HABLAR++ / HABLAR-POR-LOS-CODOS \\
72. No te cambies de chaqueta & Tú CAMBIAR-DE-OPINIÓNneg \\
73. Tienes más cara que un burro con paperas & persona caradura \\
74. Tienes menos luces que un eslabón de corcho & TENER-LA-CABEZA-VACíA
\end{tabular}

como "secundarias", debido a su escasa aplicación en este corpus: el equivalente acuñado, la descripción y el calco.

\section{El equivalente acuñado}

Después de la amplificación, la técnica más utilizada fue el equivalente acuñado. En palabras de Hurtado (2001), esta implica utilizar "un término o expresión reconocido (por el diccionario, por el uso lingüístico) como equivalente en la lengua meta" (p. 270). En la Tabla 3 se listan los ejemplos detectados.

Parece que, mientras que en las frases y locuciones proverbiales del español se emplean estructuras oracionales (Sevilla y Crida, 2013, p.112), en su traducción a la LSE se utiliza un solo signo léxico, como observamos en la revista As nosas mans (Asociación de Personas Sordas de Santiago de Compostela, 2008).

Incluimos a continuación una serie de fotogramas de los equivalentes acuñados por uso lingüístico elegidos por el alumnado (véase Figura 2). El resto de los ejemplos que aparecen en la Tabla 3 corresponden a equivalentes acuñados mediante diccionarios de LSE. En concreto, todos estos términos pueden consultarse en el DILSE: Diccionario de la lengua de signos española (Fundación CNSE, 2019). Por este motivo, ya que es un recurso accesible a través de Internet y cuenta con fotografías y vídeos que aclaran el movimiento de los diferentes signos, hemos optado por no incluir más capturas de las grabaciones del alumnado.

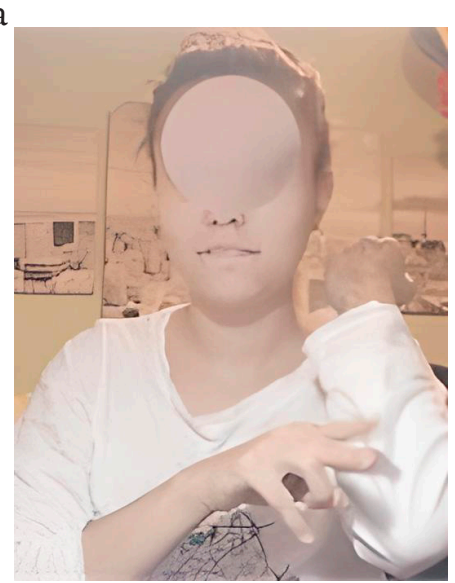

b

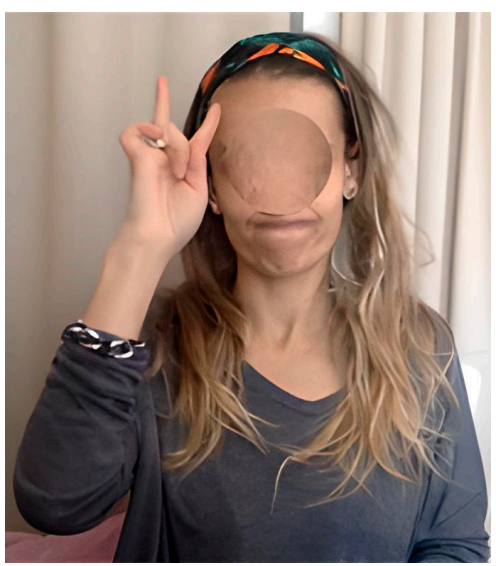

c

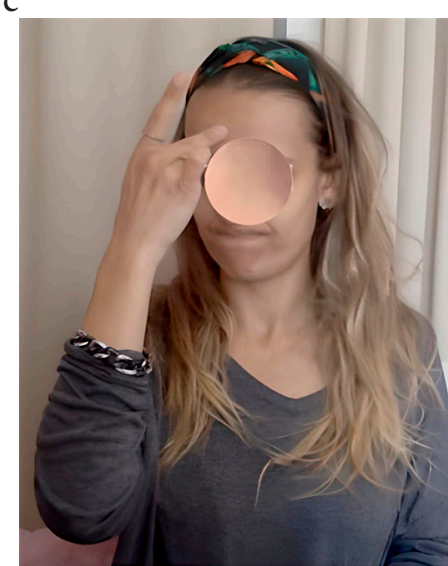

Figura 2 Ejemplos de equivalentes acuñados para la traducción de paremias a LSE. a. HABLAR-POR-LOs-CODOs; b. CAMBIAR-DE-OPINIÓN, y $c$. TENER-LA-CABEZA-VACíA. 


\section{La descripción}

Se da cuando "se reemplaza un término o expresión por la descripción de su forma y/o [sic] función" (Hurtado, 2001, p. 270). El carácter manual, gestual, espacial, icónico de la LSE, así como la simultaneidad de los articuladores, permiten que el signante pueda representar la realidad con un grado de detalle al que difícilmente llegan las lenguas orales (Herrero, 2009, p. 13).

El ejemplo localizado en nuestro corpus fue "Parece que vas pisando huevos", que se tradujo COMO PARECER TÚ CL:CAMINANDO-CON-PIESMUY-DESPACIO. En este caso, la alumna describe la forma en la que, se supone, "vas pisando huevos" (véase Figura 3). Mediante una acción esquematizada, utilizando un clasificador bimanual (palma de la mano abierta que representa ambos pies), su movimiento y la expresión facial y corporal, consiguió describir icónicamente su representación mental de esta locución.

\section{El calco}

Por último, presentamos un caso de calco como técnica de traducción: "Si no te lo digo reviento", cuya traslación a LSE fue SI YO ME-CALLOcond / AL-Final yo REVEnTaR. Según Hurtado (2001), el calco se produce cuando "se traduce literalmente una palabra o sintagma” (p. 270). Hemos

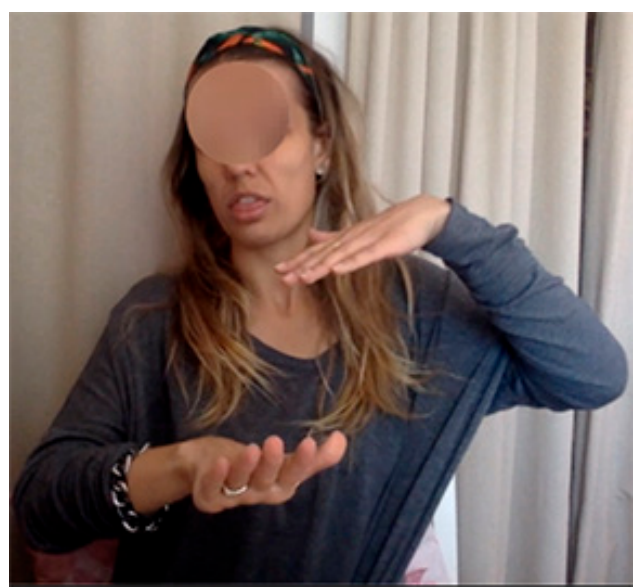

Figura 3 Acción esquematizada para describir una paremia en LSE clasificado esta traducción como calco, porque el alumno siguió la estructura condicional del original y, sobre todo, porque utilizó el signo de REVENTAR, que en este contexto no sería adecuado, por cuanto se debería haber optado por una equivalencia más dinámica, alejada del texto origen y más cercana a la cultura sorda.

\section{Conclusiones}

A partir de las diferencias culturales y lingüísticas de la comunidad sorda, en el presente trabajo analizamos cómo se puede trasladar a la LSE uno de los elementos más idiosincráticos de toda comunidad lingüística: las paremias. La traducción de estas unidades suele considerarse una de las mayores dificultades a las que se enfrenta el traductor, que utilizará las técnicas necesarias para alcanzar un equivalente funcional en la lengua meta.

En este primer acercamiento a la traducción de paremias a la LSE, y a pesar de haberse realizado con alumnado, hemos comprobado que se cumple la máxima señalada por Corpas (2003, pp. 217218) de que la paráfrasis es la principal técnica traductológica cuando existe una equivalencia nula de las UF; pero, sobre todo, hemos descubierto que esto se produce de forma independiente a la modalidad lingüística de las lenguas implicadas. Aunque la LSE es una lengua viso-gestual, utilizada por un colectivo cuya cultura se construye en clave visual, el procedimiento verbal más utilizado por este grupo de estudiantes ha sido el mismo: la amplificación (Hurtado, 2001, p. 269) o paráfrasis explicativa.

Una de las fórmulas más empleadas en este tipo de aclaraciones fue el uso de oraciones condicionales, como estructura que permite mostrar al lector sordo un ejemplo real o hipotético que facilite su comprensión de las paremias del español. Sería conveniente estudiar en la traducción paremiológica de lenguas escritas si también se aplica este tipo de proceder, o es exclusivo de las lenguas signadas. 
La última técnica reseñable es el equivalente acuñado, con la que los discentes trataron de trasvasar las paremias españolas valiéndose de un único signo léxico, incluido en diccionarios de LSE, o suficientemente reconocido por su uso en la comunidad sorda.

A lo largo de este trabajo también hemos detectado ciertas carencias sobre esta materia en las lenguas signadas: la falta de publicaciones científicas, la pobreza en la concreción de la competencia fraseológica según los niveles del MCER, la necesidad de que se desarrollen materiales didácticos específicos para la adquisición de esta competencia, o una mayor presencia de la paremiología en la formación de futuros traductores e intérpretes de LSE. Con el presente artículo, hemos pretendido hacer patente la importancia que tiene esta instrucción específica en traducción paremiológica durante el proceso formativo de estos profesionales en ciernes.

Somos conscientes de las limitaciones de este trabajo, por su diseño metodológico y el corpus utilizado, pero como primera aproximación a la traducción paremiológica puede ser válido. De cara a un futuro, sería necesario superar el nivel léxico analizado y ahondar en cómo se realiza la traducción de paremias a la LSE a nivel textual y discursivo, valorar cómo resuelven este tipo de estructuras los intérpretes de LSE profesionales, así como determinar las características propias de las UF de la LSE - si es que existen tal y como las entendemos en las lenguas orales-. Coincidimos con Corpas en que "[1]a ampliación de lenguas de estudio, corrientes de investigación y enfoques novedosos implican necesariamente un replanteamiento continuo de las bases de las teorías fraseológicas actuales" (Corpas y Ortiz, 2017, p. 277), y creemos que las lenguas de signos tienen mucho que aportar a la fraseología y a la traducción fraseológica y paremiológica.

\section{Referencias}

Asociación de Personas Sordas de Santiago de Compostela (2008). As nosas mans. Grafisant. https://bit. ly/3nmwAvT
Cabeza-Pereiro, M. C. y Ramallo, F. (2016). Lenguas de signos y educación en España: una aproximación desde la comunidad sorda. Language Problems \& Language Planning, 40(1), 1-25. https://doi. org/10.1075/lplp.40.1.01cab

Centro de Normalización Lingüística de la Lengua de Signos Española (CNLSE). (2014). La lengua de signos española hoy. Informe de la situación de la lengua de signos española. Actas del Congreso CNLSE sobre la Investigación de la Lengua de Signos Española. Real Patronato sobre Discapacidad. https://bit. ly/2GGx5QY

Cobeta, M. M. (2000). En torno a la traducción de paremias. En M. L. Casal, G. Conde, J. Lago, L. Pino y N. Rodríguez (Coord.), La lingüistica francesa en España camino del siglo XXI (Vol. 1, pp. 263-270). Arrecife.

Corpas, G. (2003). Diez años de investigación en fraseología: análisis sintáctico-semánticos, contrastivos y traductológicos. Iberoamericana.

Corpas, G. (2013). Detección, descripción y contraste de las unidades fraseológicas mediante tecnologías lingüísticas. En I. Olza y E. Manero (Eds.), Fraseopragmática (pp. 335-373). Frank \& Timme.

Corpas, G. y Ortiz, M. L. (2017). Fraseología y paremiología: una entrevista con Gloria Corpas Pastor. ReVEL, 15(29), 271-280. http://www.revel.inf.br/ files/59c0f4bc2bb047821269aa0981caa08e.pdf

Costello, B., Fernández, J., Villameriel, S. y Mosella, M. (2012). Una lengua sin nativos: consecuencias para la normalización. En Estudios sobre la lengua de signos española. III Congreso Nacional de la lengua de signos española. Hacia la normalización de un derecho lingüistico y cultural (pp. 371-388). UNED/CNSE.

De Meulder, M. y Murray, J. J. (2017). Buttering their bread on both sides? The recognition of sign languages and the aspirations of deaf communities. Language Problems and Language Planning, 41(2), 136-158. https://doi.org/10.1075/lplp.41.2.04dem

Díaz, A. M. y Sabio, J. A. (2017). Aplicaciones a la enseñanza de la traducción del mínimo paremiológico del portugués. Caracol, (14), 104-129. https://doi. org/10.11606/issn.2317-9651.v0i14p104-129

España, Ley 27/2007, por la que se reconocen las lenguas de signos españolas y se regulan los medios de apoyo a la comunicación oral de las personas sordas, con discapacidad auditiva y sordociegas. 24 de octubre de 2007. Boletín Oficial del Estado, n. ${ }^{\circ} 255$ (octubre 23). https://www.boe.es/eli/es/1/2007/10/23/27 
España, Ley Orgánica 3/2018, de protección de datos personales y garantía de los derechos digitales. 6 de diciembre de 2018. Boletín Oficial del Estado, n. ${ }^{\circ} 294$ (diciembre 5). https://www.boe.es/eli/es/ lo/2018/12/05/3

Esteban, M. L. y Ramallo F. (2019). Derechos lingüísticos y comunidad sorda: claves para entender la minorización. Revista de Estudios de Lenguas de Signos REVLES: Aspectos lingüisticos y de adquisición de las lenguas de signos, (1), 20-52. http://www.revles.es/ index.php/revles/article/view/19

Flick, U. (2015). El diseño de investigación cualitativa. Ediciones Morata.

Fundación CNSE (2019). DILSE: Diccionario de la lengua de signos española. https://fundacioncnse-dilse.org

González-Montesino, R. H. y Saavedra-Rodríguez, S. (2020). La traducción de las metáforas del vino a la lengua de signos española. En M. Ibáñez (Ed.), Enotradulengua: vino, lengua y traducción (pp. 221248). Peter Lang. https://doi.org/10.2307/j. ctv 1 ks0ggj.14

Herrero, Á. (2009). Gramática didáctica de la lengua de signos española (LSE). Fundación CNSE/SM.

Hurtado, A. (2001). Traducción y traductología. Introducción a la traductología. Cátedra.

Instituto Nacional de Estadística. (2008). Encuesta sobre discapacidades, autonomia personal y situaciones de dependencia. https://bit.ly/3jMxCzb

Jordán, F. (2010). Dichos y refranes populares. Cangilón, (33), 323-331. http://cangilon.regmurcia.com/revista/N33/N33-26.pdf

Kusters, A., De Meulder, M. y O’Brien, D. (2017). Innovations in deaf studies. Critically mapping the field. En A. Kusters, M. De Meulder y D. O’Brien (Eds.), Innovations in deaf studies: The role of deaf scholars (pp. 1-56). Oxford University Press.

Leeson, L., Van den Bogaerde, B., Rathmann, C. y Haug, T. (2016). PRO-Sign. Sign languages and the Common European Framework of Reference for Languages. European Centre for Modern Languages of the Council of Europe.

Leigh, I. y Andrews, J. (2017). Deaf people and society: Psychological, sociological, and educational perspectives. Routledge. https://doi. org/10.4324/9781315473819

Martín, J. M. (2008). El grado de conocimiento de las unidades fraseológicas en estudiantes de Traducción e Interpretación. Paremia, (17), 189-199. https://cvc. cervantes.es/lengua/paremia/pdf/017/017_martin. pdf

Massone, M. I., Druetta, J. C. y Simón, M. (2003). Arquitectura de la escuela de sordos. Libros en Red.

Neunzig, W. y Tanqueiro, H. (2007). Estudios empiricos en traducción: enfoques y métodos. Documenta Universitaria.

PACTE (2003). Building a translation competence model. En F. Alves (Ed.), Triangulating translation (pp. 4366). John Benjamins. https://doi.org/10.1075/ btl.45.06pac

Padden, C. (1980). The deaf community and the culture of deaf people. En C. Baker y R. Battison (Eds.), Sign language and the deaf community: Essays in honor of William Stokoe (pp. 89-103). National Association of the Deaf.

Parlamento Europeo y Consejo de la Unión Europea (2016). Reglamento UE 679/2016 del Parlamento Europeo y del Consejo, relativo a la protección de las personas físicas en lo que respecta al tratamiento de datos personales y a la libre circulación de estos datos. 4 de mayo de 2016. Diario Oficial de la Unión Europea, L 119 (abril 27). https://www.boe.es/ doue/2016/119/L00001-00088.pdf

Pöchhacker, F. (2004). Introducing interpretingstudies. Routledge. https://doi.org/10.4324/9780203504802

Quadros, R. y Segala, R. (2015). Tradução intermodal, intersemiótica e interlinguística de textos escritos em Português para a Libras oral. Cadernos de Tradução, 35(2), 354-386. https://doi. org/10.5007/2175-7968.2015v35nesp2p354

Quadros, R. y Souza, S. (2008). Aspectos da tradução/encenação na língua de sinais brasileira para um ambiente virtual de ensino: práticas tradutórias do curso de letras libras. Em R. Quadros (Org.), Estudos Surdos III (pp. 168-207). Editora Arara Azul.

Richart, M. (2008). Las unidades fraseológicas y su resistencia a la traducción. Foro de profesores de E/ $L E$, (4), 1-10. https://dialnet.unirioja.es/servlet/ articulo codigo $=4896388$

Rodríguez, M. A. (1992). Lenguaje de signos. Confederación Nacional de Sordos de España, Fundación ONCE.

Segala, R. (2010). Tradução intermodal e intersemiótica/interlinguistica: portugués escrito para a lingua de sinais [Tesis de maestría, Universidade Federal de Santa Catarina]. Repositorio Institucional UfSC. https:// bit.ly/30Sz8Zb 
Sevilla, J. y Crida, C. (2013). Las paremias y su clasificación. Paremia, (22), 105-114. https://cvc.cervantes.es/ lengua/paremia/pdf/022/009_sevilla-crida.pdf

Sevilla, J. y Sevilla, M. (2005, 2 de septiembre). La traducción de paremias populares: el refrán y la frase proverbial. El Trujamán. https://bit.ly/2GHHFXV

Simons, H. (2011). El estudio de caso: teoria y práctica. Ediciones Morata.
Storch, J. G. (1998). El nombre de nuestra lengua [Sesión de conferencia]. 1. ${ }^{\text {er }}$ Congreso Ibero-Americano de Educación Bilingüe para Sordos, Lisboa, Portugal. https://eprints.ucm.es/id/eprint/62622/14/signa. pdf

Timofeeva, L. (2012). Sobre la traducción fraseológica. ELUA, Estudios de Lingüistica, (26), 405-432. https://doi.org/10.14198/ELUA2012.26.15

\section{Anexo}

\begin{tabular}{|c|c|}
\hline & Sistema de glosas utilizado para transcribir las traducciones a LSE \\
\hline SIGNO & Glosa utilizada para cada signo léxico. Representa su significado base, no marcado (p. ej. PeRsona) \\
\hline \multirow[t]{2}{*}{ Cl: } & $\begin{array}{l}\text { Glosa para representar los clasificadores. Todo lo que sigue y que se separa entre guiones representa el significado } \\
\text { de dicha construcción. }\end{array}$ \\
\hline & p. ej. CL:CAMINANDO-CON-PIES-MUY-DESPACIO \\
\hline \multirow[t]{2}{*}{ SIGNO-SIGNO } & Dos palabras (o más) son necesarias para reproducir el significado de un solo signo. \\
\hline & p. ej. CAMBIAR-DE-OPINIÓN \\
\hline \multirow[t]{3}{*}{ SIGNO-me/te } & Representa los participantes de una acción, especialmente para verbos direccionales con dos concordancias \\
\hline & (sujeto-receptor). \\
\hline & p. ej. DAR-me - 'tú me das' \\
\hline \multirow[t]{2}{*}{ SIGNO++ } & Con ++ se especifica el número, mediante el procedimiento de repetición. \\
\hline & p. ej. PERSONA ++ \\
\hline$\underline{\text { SIGNO }}$ & Se subrayan todos los signos acompañados por componentes no manuales \\
\hline \multirow[t]{2}{*}{ SIGNot } & Es la forma con la que se tematiza la información. Se marca mediante la elevación de las cejas y una pequeña pausa. \\
\hline & p. ej. Iút BUENO \\
\hline \multirow[t]{2}{*}{ SIGNO"signo" } & Entre comillas se especifica mediante componentes hablados (vocalización) el signo articulado. \\
\hline & p. ej. ABURRIR no"no me apetece" \\
\hline \multirow[t]{3}{*}{$\underline{\text { SIGNoret }}$} & Se utiliza en las preguntas retóricas o preguntas polarizadas. Supone una elevación de las cejas, inclinación de la \\
\hline & cabeza y cuerpo hacia delante y mirada al interlocutor. \\
\hline & p. ej. DAR-me Quéret \\
\hline \multirow[t]{2}{*}{$\underline{\text { SIGNOafirm }}$} & Es para indicar cuando se enfatiza una oración afirmativa con el asentimiento de cabeza. \\
\hline & p. ej. AHÍ HABERafirm \\
\hline \multirow[t]{2}{*}{ SIGNoneg } & Indica oraciones negativas, principalmente mediante la negación con la cabeza. \\
\hline & p. ej. INTERESAR Noneg \\
\hline \multirow[t]{2}{*}{$\underline{\text { SIGNOCOnd }}$} & Oraciones condicionales. Supone la elevación de las cejas y cierto movimiento o adelantamiento de la cabeza. \\
\hline & p. ej. SI TÚ INTENTARCond \\
\hline \multirow[t]{2}{*}{ SIGNO / 0 // } & Una sola barra significa una pausa breve, y dos, una pausa más larga \\
\hline & p. ej. SI DUDARCond / PREGUNTARafirm \\
\hline \multirow[t]{3}{*}{ SIGNO $[\log X]$} & Marca localizaciones (izquierda/derecha), es decir, se articula un signo en un lugar que no le corresponde para usar el \\
\hline & espacio gramatical o to \\
\hline & p. ej. SUERTE[loq izq] \\
\hline
\end{tabular}

Cómo citar este artículo: González- Montesino, R. H. (2022). Aproximación a la traducción de unidades paremiológicas del español a la lengua de signos española. Íkala, Revista de Lenguaje y Cultura, 27(1), 31-48. https://doi.org/10.17533/udea.ikala.v27n1a02 